\title{
Flesh Color Inheritance and Gene Interactions among Canary Yellow, Pale Yellow, and Red Watermelon
}

\author{
Haejeen Bang \\ Vegetable and Fruit Improvement Center, Department of Horticultural Sciences, \\ Texas A\&M University, College Station, TX 77843
}

Angela R. Davis

U.S. Department of Agriculture, Agricultural Research Service, South Central Agricultural

Research Laboratory, P.O. Box 159, Highway 3 West, Lane, OK 74555

\author{
Sunggil Kim ${ }^{1}$ \\ Vegetable and Fruit Improvement Center, Department of Horticultural Sciences, \\ Texas A\&M University, College Station, TX 77843
}

Daniel I. Leskovar

Vegetable and Fruit Improvement Center, Department of Horticultural Sciences, Texas A\&M

University, College Station, TX 77843; and Texas AgriLife Research, Texas A\&M

University, 1619 Garner Field Road, Uvalde, TX 78801

\author{
Stephen R. King ${ }^{2}$ \\ Vegetable and Fruit Improvement Center, Department of Horticultural Sciences, \\ Texas A\&M University, College Station, TX 77843
}

\begin{abstract}
AdDITIONAL INDEX wORDs. lycopene $\beta$-cyclase, biosynthesis, carotenoid profile, molecular marker
Abstract. Two loci, $C$ and $i-C$, were previously reported to determine flesh colors between canary yellow and red watermelon (Citrullus lanatus). Recently, lycopene $\beta$-cyclase (LCYB) was found as a color determinant gene for canary yellow $(C)$ and a codominant cleaved amplified polymorphic sequence (CAPS) marker was developed to identify canary yellow and red alleles. The inhibitor of canary yellow $(i-C)$, as reported in a previous work, was not detected in our original family derived from a cross between canary yellow and red parents. To identify additional genetic determinants such as $i$ - $C$, we prepared a new family using 'Yellow Doll' (canary yellow) and 'Sweet Princess' (red), which was reported to carry the inhibitor gene $i-C$ as parents. A new distinct class of flesh color, pale yellow, was identified in the progeny from the new canary yellow $\times$ red cross. The predominant carotenoid in canary yellow and pale yellow phenotypes was neoxanthin, followed by violaxanthin and neochrome; pale yellow contained less total carotenoids, but had more minor carotenoids compared with canary yellow. The chi-square goodness-of-fit test indicated that there are two genes involved in determining flesh color among canary yellow, pale yellow, and red, but the segregation pattern did not fit the pattern as reported for an $i$ - $C$ gene. When the genotype of the family 'Yellow Doll' $\times$ 'Sweet Princess' was analyzed with our LCYB CAPS marker, the flesh color of every individual perfectly cosegregated with the marker. The new pale yellow phenotype also cosegregated with the marker linked to the $C$ allele, indicating that the recessive $p y$ phenotype (pale yellow) must carry at least one of the $C$ alleles for expression. Therefore, we propose to designate $p y$ for a pale yellow determinant along with $C$ as a canary yellow determinant. A homozygous recessive $p y$ gene resulted in pale yellow flesh color in the presence of a dominant $C$.
\end{abstract}

\footnotetext{
Received for publication 1 Apr. 2010. Accepted for publication 10 May 2010. This material is based upon work supported by the Cooperative State Research, Education, and Extension Service, U.S. Department of Agriculture under Agreements No. 2004-34402-14768, 2005-34402-16401, and 2006-3440217121, "Designing Foods for Health," through the Vegetable and Fruit Improvement Center, Texas AgriLife Research. We acknowledge Drs. Monica Menz and Eun-Gyu No from the Laboratory for Plant Genome Technology, Texas A\&M University, for technical advice, and Michael Faries, Amy Helms, and Sheila Magby for technical support.

${ }^{1}$ Currrent address: Department of Plant Biotechnology, Biotechnology Research Institute, Chonnam National University, Gwangju 500-757, South Korea.

${ }^{2}$ Corresponding author. E-mail: srking@tamu.edu.
}

Flesh color is an important fruit quality trait that helps to determine attractiveness and is indicative of the potential health benefits of watermelon. The coloration of watermelon flesh is attributable to its carotenoid composition and content. Carotenoids are bioactive compounds with antioxidant properties that may reduce the risk of certain cancers and cardiovascular diseases (Bramley, 2000; Gerster, 1997; Giovannucci et al., 2002). Flesh color in watermelon has been categorized as red, canary yellow, salmon yellow, orange, and white flesh; red flesh has recently been reclassified into two distinct flesh colors, coral red and scarlet red (Gusmini and Wehner, 2006).

Several researchers have previously reported flesh color inheritance in watermelon (Henderson, 1989; Henderson et al., 1998; Poole, 1944; Porter, 1937; Shimotsuma, 1963), which has 
resulted in a genetic series summarized in Wehner (2007). In this series, canary yellow $(C)$ is dominant to other colors $(c)$ except white $(W f)$, which is reported to be epistatic to yellow flesh $(B)$, where genotype $W f_{-} B_{-}$or $W f_{-} b b$ was white fleshed, $w f w f B_{\text {_ }}$ was yellow fleshed, and $w f w f \bar{b} b$ was red fleshed; the gene for coral red flesh $(Y)$ is a member of a multiple allelic system at that locus where coral red flesh $(Y)$ is dominant to orange flesh $\left(y^{O}\right)$ and salmon yellow $(y)$, and orange flesh $\left(y^{O}\right)$ is dominant to salmon yellow $(y)$; canary yellow flesh $(C)$ has also been reported to be epistatic to $Y$ and influenced by a inhibitor of canary yellow $(i-C)$ where homozygous recessive $i-C$ alleles results in red flesh even in the presence of $C$ (Henderson et al., 1998). Despite this reported series of genes, there is still much confusion regarding the genetics of flesh coloration in watermelon. One reason may be that flesh colors can be ambiguous, since the "yellow" referred to by Shimotsuma (1963) was not defined as canary or salmon, which are now considered distinct colors, nor was the white flesh in this same report characterized as being a lack of carotenoids (a characteristic of certain citron watermelons), or containing low levels of light-colored or colorless carotenoids. The genotypes used in previous reports were often breeding lines or sometimes $F_{1}$ hybrids, making it difficult to re-examine segregation patterns or confirm the colors used in the reports. These earlier reports on flesh color in watermelon also lacked carotenoid profiles and molecular data to assist in identifying the genetics of the different flesh colors.

Carotenogenesis and its regulating mechanisms have been comprehensively investigated in tomato (Solanum lycopersicum), and several of these studies have included biochemical and molecular characterization of the pathway and genes (Hirschberg, 2001; Isaacson et al., 2002, 2004; Ronen et al., 1999, 2000; Sauret-Güeto et al., 2003). More recently, flesh color mutant comparisons between tomato and watermelon were made (Lewinsohn et al., 2005a, 2005b), but these comparisons lacked accurate genetic inheritance or functional analysis of watermelon genes and were based solely on carotenoid profiles. Moreover, unlike the cases for tomato mutants, the identities of predominant carotenoids in several watermelon color mutants were not fully established (Isaacson et al., 2002; Lewinsohn et al., 2005a, 2005b; Ronen et al., 2000; Tadmor et al., 2004, 2005).

Lycopene $\beta$-cyclase (LCYB) is a critical enzyme participating in the cyclization of lycopene into $\beta$-carotene via $\gamma$-carotene in the carotenoid biosynthetic pathway. There are two known forms of LCYB in plants: chromoplast specific and chloroplast specific. In tomato, chromoplastic-specific LCYB (CYCB) was reported as the form of cyclase responsible for coloration during fruit ripening (Hirschberg, 2001; Ronen et al., 2000). Wild-type tomato is reported to be red in color resulting from a mutation in CYCB. The carotenoid profile of red tomato is very similar to that of red watermelon (Lewinsohn et al., 2005a, 2005b; Tadmor et al., 2004, 2005). However, to fully determine the genetic regulation of flesh color, genetic data along with biochemical profiles should be studied together.

Recently, the flesh color inheritance of canary yellow and red watermelon was conducted for molecular marker development using biochemical information and gene sequence data, as well as flesh color segregation patterns (Bang et al., 2007). This report identified a single dominant gene for canary yellow and a cleaved amplified polymorphic sequence (CAPS) marker was developed based on a single-nucleotide polymorphism (SNP) within the LCYB gene; the resultant CAPS marker has been used to reliably distinguish canary yellow from red flesh in numerous populations (S. King, unpublished data). However, this report did not identify any effect of an $i-C$ gene as reported by Henderson et al. (1998), which could be because the parents used in the Bang et al. (2007) study differed from those used in Henderson et al. (1998).

In this work, we validate the finding of our previous inheritance study showing that a single gene distinguishes canary yellow and red watermelon using parents reported to carry recessive $i$ - $C$ alleles and we confirm that the previously developed codominant CAPS marker can reliably distinguish between canary yellow and red flesh in watermelon. We also report a new flesh color category with a unique carotenoid profile, and provide genetic, biochemical, and molecular evidence of flesh color inheritance among three flesh colors in watermelon.

\section{Materials and Methods}

Plant materials. Because 'Sweet Princess' watermelon was previously reported to carry homozygous recessive inhibitory $(i-C)$ alleles (reported genotype: $c c Y Y i-C i-C)$ and 'Yellow Doll' watermelon was reported to carry homozygous dominant $(I-C)$ alleles (reported genotype: CC YY I-CI-C) (Henderson et al., 1998), these parents were used as the red and canary yellow-fleshed parents, respectively; $\mathrm{F}_{1}, \mathrm{~F}_{2}$, and backcross (BC) populations were generated by controlled pollinations in the greenhouse. $\mathrm{F}_{3}$ families were also created from selected $F_{2}$ plants. Seeds were sown in flats and 5- to 6week-old seedlings were transplanted into 8-L pots containing Pro-Mix BX growing medium (Premier Horticulture, Quakertown, PA). All plants were irrigated multiple times daily and were supplemented with a general purpose $20 \mathrm{~N}-8.8 \mathrm{P}-16.6 \mathrm{~K}$ fertilizer (Scotts, Marysville, $\mathrm{OH}$ ) weekly. All fruit for evaluation were harvested at full maturity between 45 to $50 \mathrm{~d}$ after pollination. Harvested fruit were cut longitudinally, photographed, and categorized as yellow or red. Flesh samples were taken from the heart tissue and stored at $-80^{\circ} \mathrm{C}$ for high-performance liquid chromatography (HPLC) analysis. Leaf tissues were collected and stored individually at $-20{ }^{\circ} \mathrm{C}$ for genotype analysis.

Statistical ANALYSIS. The number of genes involved in color determination for each cross was estimated by the chisquare goodness-of-fit test using fruit color characterizations.

Carotenoid extraction and HPLC anAlysis For PROFILING. Frozen samples were thawed and then homogenized. Canary yellow and pale yellow flesh samples from $\mathrm{F}_{2}$ progeny were concentrated to 10 times their original volume using centrifugation at $10,000 g_{n}$ for $15 \mathrm{~min}$ at $4{ }^{\circ} \mathrm{C}$. Carotenoid extraction from red flesh was carried out without concentrating the sample. One gram of each sample was extracted with $10 \mathrm{~mL}$ of hexane (Fish et al., 2002) and $100 \mu \mathrm{L}$ of extract $(9.1 \mu \mathrm{g}$ fresh weight of red and $91 \mu \mathrm{g}$ fresh weight of canary yellow and pale yellow) was injected for HPLC analysis.

In a separate experiment, each sample was extracted using a modified ethanol method (Rodriguez, 2001) to identify polar carotenoids that are only slightly miscible in hexane. Briefly, $1 \mathrm{~g}$ of extract was polytroned in $9 \mathrm{~mL}$ of $95 \%$ ethanol. The samples were briefly polytroned, sonicated for $15 \mathrm{~min}$, then centrifuged for $15 \mathrm{~min}$ at $7000 g_{\mathrm{n}}$ at $4{ }^{\circ} \mathrm{C}$. The supernatant was collected, $3 \mathrm{~mL}$ of ethanol was added, and the sample was polytroned and then centrifuged as above. Five milliliters of the supernatant was filtered using $0.45-\mu \mathrm{m}$ polytetrafluoroethylene syringe filters (Daigger, Vernon Hills, IL) and dried to $1.5 \mathrm{~mL}$ 
under nitrogen. The equivalent of $26 \mu \mathrm{g}$ fresh weight of extract per milliliter was injected for HPLC analysis.

Polytroned samples of canary yellow and pale yellow were sent to Craft Technologies (Wilson, NC) for accurate determination of carotenoids using previously published methodology (Craft, 2001). About $5 \mathrm{~g}$ of each sample was extracted three times with 50:50 methanol/tetrahydrofuran (THF) to obtain a complete carotenoid extraction. The methanol/THF extract was back extracted, after the addition of water, into hexane until colorless. Samples of the hexane extract were dried in a SpeedVac ${ }^{\mathrm{TM}}$ (Thermo Savant, Holbrook, NY) and resuspended to an appropriate concentration in a small volume of ethyl acetate and then diluted with the mobile phase. Sample extracts were scanned on a spectrophotometer to estimate total carotenoids using $2500 \mathrm{~g} \cdot \mu \mathrm{g}^{-1} \cdot \mathrm{cm}^{-1}$ as the absorptivity. Portions of sample extracts were saponified using $\mathrm{KOH}$ and pyrogallol as an antioxidant. All samples were separated using a $\mathrm{C}_{30}$ HPLC gradient with diode array detection.

CAPS MARKER ANALYSIS. CAPS marker analysis was carried out as described in Bang et al. (2007). A total of 210 individual plants were analyzed by LCYB genotyping and included both parents, $\mathrm{F}_{1}, \mathrm{~F}_{2}$, BC (both parents), and $\mathrm{F}_{3}$ progenies.

\section{Results and Discussion}

Flesh COlOR InHeritance. Our previous study using PIs (PI accessions) as parents determined that a single watermelon LCYB gene was the determinant of canary yellow or red flesh color production in the above population (Bang et al., 2007). This was contradictory to the two-gene model proposed by Henderson et al. (1998), and could be due to the parental PI accessions, which appear to lack the recessive inhibitory gene $i-C$. To identify additional genetic determinants of flesh colors, such as $i-C$, we prepared a new family using 'Yellow Doll' $\times$ 'Sweet Princess', which were used to develop the two-gene model where 'Sweet Princess' is homozygous recessive for the $i$ - $C$ alleles.

Upon visual observation, the flesh color produced in this population was classified into three categories. These included not only red and canary yellow, but also coloration we termed "pale yellow." The pale yellow category was termed due to its off-white appearance with a light yellow tint (Fig. 1). We had noted this color in previous populations created between canary yellow and red, but considered that they were immature canary yellow because they came from field-grown plants without dated pollination or known maturity. It was unlikely that the pale yellow was due to immaturity; all plants in this population were grown in the greenhouse using dated pollinations, and all fruit were harvested at least $45 \mathrm{~d}$ after pollination. This pale yellow color was very similar to 'Cream of Saskatchewan', which has been categorized as having white flesh (Gusmini and Wehner, 2006), although the color is distinct from the pure white flesh of citron watermelon.

To establish the number of mutations that determine flesh color of this population, we attempted to fit the observed segregation patterns to various genetic models. First, the two gene-color model that predicts the presence of $C$ and $i$ - $C$ genes was used (Table 1). Here, we grouped canary yellow and pale
Table 1. Chi-square test of the segregation ratio between yellow and red colors of watermelon fruit in the $F_{2}$ and backcross progeny derived from a cross between 'Yellow Doll' (canary yellow) and 'Sweet Princess' (red).

\begin{tabular}{lcccc}
\hline Generation & $\begin{array}{c}\text { Observed } \\
\text { (yellow:red) }^{z}\end{array}$ & $\begin{array}{c}\text { Expected } \\
\text { (yellow:red) }\end{array}$ & $\chi^{2}$ & $P$ \\
\hline $\mathrm{F}_{1}$ & $10: 0$ & $1: 0$ & 0 & 1 \\
$\mathrm{~F}_{2}$ & $69: 19$ & $3: 1$ & 0.55 & 0.46 \\
& & $9: 7$ & 17.56 & $<0.01$ \\
$\mathrm{BC}_{\text {Canary Yellow }}$ & $17: 0$ & $1: 0$ & 0 & 1 \\
$\mathrm{BC}_{\text {Red }}$ & $18: 21$ & $1: 1$ & 0.23 & 0.63 \\
& & $1: 3$ & 9.31 & $<0.01$ \\
\hline
\end{tabular}

${ }^{\mathrm{z} Y e l l o w}=$ canary yellow + pale yellow.

yellow together as "yellow" according to the two gene-color model between yellow and red watermelon. Statistical analysis of color segregation indicated that the observed data did not significantly deviate from $3: 1$ for $F_{2}$ and $1: 1$ for the $B C$ (red parent) at $P=0.05$. These results indicate that a single gene determines the color between yellow (canary yellow and pale yellow) and red. In contrast, the observed data of the $\mathrm{F}_{2}$ progeny was not a good fit to $9: 7$ or that of BC (red parent) to 1:3, which were previously observed by Henderson et al. (1998). We did not observe an $i-C$ gene effect as reported by Henderson et al. (1998).

Next, we determined whether a mutation other than $i-C$ was responsible for the pale yellow flesh color. For this purpose, individuals with yellow and the pale yellow colors were scored as separate categories (Table 2). Test results showed epistatic gene interactions that fit to a 9:3:4 segregation ratio. This result demonstrates that there are two loci involved in coloration among canary yellow, pale yellow, and red watermelon. Namely, pale yellow fit a model for a single recessive gene influenced by the presence of $C$, which is distinct from the previously reported $i-C$ gene because a recessive $i-C$ gene would lead to red flesh.

Interesting color pattern in the flesh was identified, consisting of mixed colors of yellow and red swirled together in separate sectors or in mixed patterns in the heart and placental tissues. We classified these mixed colors as red if the flesh showed more than $50 \%$ area with red in cross section. This cannot be described as an inhibitor gene effect because the frequency was very low $(6 \%$ in this population) and it did not fit an $i-C$ gene segregation pattern. Henderson et al. (1998) also reported this phenotype in their population and proposed a modifier gene.

Carotenoid profiles. To establish biochemical basis for the flesh color variations in our population, we determined carotenoid profiles for each flesh color. Because it is well 
established that the predominant carotenoid in red watermelon is lycopene (Perkins-Veazie et al., 2006), which is fully soluble in hexane, this solvent was used to extract the red flesh samples. Hexane was also used on all samples, but ethanol was additionally used to verify and quantify polar carotenoids in canary yellow and pale yellow watermelon samples. Additionally, a methanol/THF extraction method was used to verify our ethanol results of the canary yellow and pale yellow samples. The carotenoid profiles of the extracted samples were determined by combining in-house HPLC analyses and verification of select samples by Craft Technologies.

The predominant carotenoid in the red samples was, as expected, lycopene (Table 3), with a total carotenoid concentration of $36 \mu \mathrm{g} \cdot \mathrm{g}^{-1}$ fresh weight. Xanthophylls were in low abundance in canary yellow and pale yellow and thus were difficult to identify using conventional HPLC methodology. To distinguish xanthophylls, high-resolution HPLC (HR-HPLC) analysis data were obtained using the carotenoid profiling service from Craft Technologies, Inc.

HR-HPLC analyses of canary yellow and pale yellow extracts revealed numerous hydrocarbon carotenes that were determined to be xanthophyll esters (Fig. 2A); saponification was therefore needed to clarify the predominant carotenoids in canary yellow and pale yellow flesh watermelon. The total carotenoid content (by HPLC) of the saponified canary yellow sample was determined to be $2.73 \mu \mathrm{g} \cdot \mathrm{g}^{-1}$ fresh weight, while the

Table 2. Chi-square test of the segregation ratio among canary yellow (CY), pale yellow (PY), and red colors of watermelon fruit in the $F_{2}$ and backcross progeny derived from a cross between 'Yellow Doll' (canary yellow) and 'Sweet Princess' (red).

\begin{tabular}{lccll}
\hline Generation & $\begin{array}{c}\text { Observed } \\
\text { (CY:PY:red) }\end{array}$ & $\begin{array}{c}\text { Expected } \\
\text { (CY:PY:red) }\end{array}$ & \multicolumn{1}{c}{$\chi^{2}$} & \multicolumn{1}{c}{$P$} \\
\hline $\mathrm{F}_{1}$ & $10: 0: 0$ & $1: 0: 0$ & 0 & 1 \\
$\mathrm{~F}_{2}$ & $51: 18: 19$ & $9: 3: 4$ & 0.59 & 0.74 \\
$\mathrm{BC}_{\mathrm{CY}}$ & $17: 0: 0$ & $1: 0: 0$ & 0 & 1 \\
$\mathrm{BC}_{\text {red }}$ & $8: 10: 21$ & $1: 1: 2$ & 0.44 & 0.80 \\
\hline
\end{tabular}

pale yellow sample contained $0.70 \mu \mathrm{g} \cdot \mathrm{g}^{-1}$ fresh weight. The predominant carotenoid in canary yellow and pale yellow flesh after saponification was neoxanthin (Table 3, Fig. 2B), with canary yellow containing $1.66 \mu \mathrm{g} \cdot \mathrm{g}^{-1}$ fresh weight $(61 \%$ of total) and pale yellow containing $0.29 \mu \mathrm{g} \cdot \mathrm{g}^{-1}$ fresh weight $(41 \%$ of total). Violaxanthin and neochrome were also identified as relatively major carotenoids present in canary yellow and pale yellow flesh, each making up over $10 \%$ of the total carotenoids in each of these flesh colors (Table 3 ). Other carotenoids indentified in samples of canary yellow and pale yellow flesh include trace amounts $(<5 \%$ of total) of phytoene/phytofluene and $\beta$-carotene. Pale yellow flesh also contained small amounts of $\zeta$-carotene, lycopene (trans and cis), $\gamma$-carotene, and $\beta$-cryptoxanthin. It should be pointed out that the pale yellow samples were more concentrated due to the lower carotenoid content and some of the very low peaks in Fig. 2B may have not been visible in the less concentrated canary yellow samples; these carotenoids are identified in Table 3 as "detected" to distinguish them from the more obvious peaks that differentiate pale yellow from canary yellow. There were also a number of unknown peaks (Fig. 2B), with pale yellow flesh containing more unknown carotenoids than canary yellow flesh, but again, some of these may have been an artifact of the higher concentration of the pale yellow flesh.

Previous results in our laboratory have demonstrated that no carotenoids were detectable in pure white flesh watermelon samples such as found in citron watermelon. We ran samples of the pure white flesh PI494531 using a spectrophotometric method that does not require extraction of a sample (Davis et al., 2007) and found it to contain no detectable carotenoids (data not shown) to distinguishes it from the pale yellow flesh in this study.

INHERITANCE OF CAROTENOID PROFILES. Red-fleshed watermelon have high concentrations of lycopene, which impart the red color. Canary yellow flesh can be expected when the LCYB gene is functional in a functional carotenoid pathway. This leads to the conversion of upstream carotenoids into downstream compounds, with neoxanthin as the primary carotenoid accumulated, and violaxanthin and neochrome (products

Table 3. Carotenoids detected after saponification using HPLC in three flesh colors of watermelon.

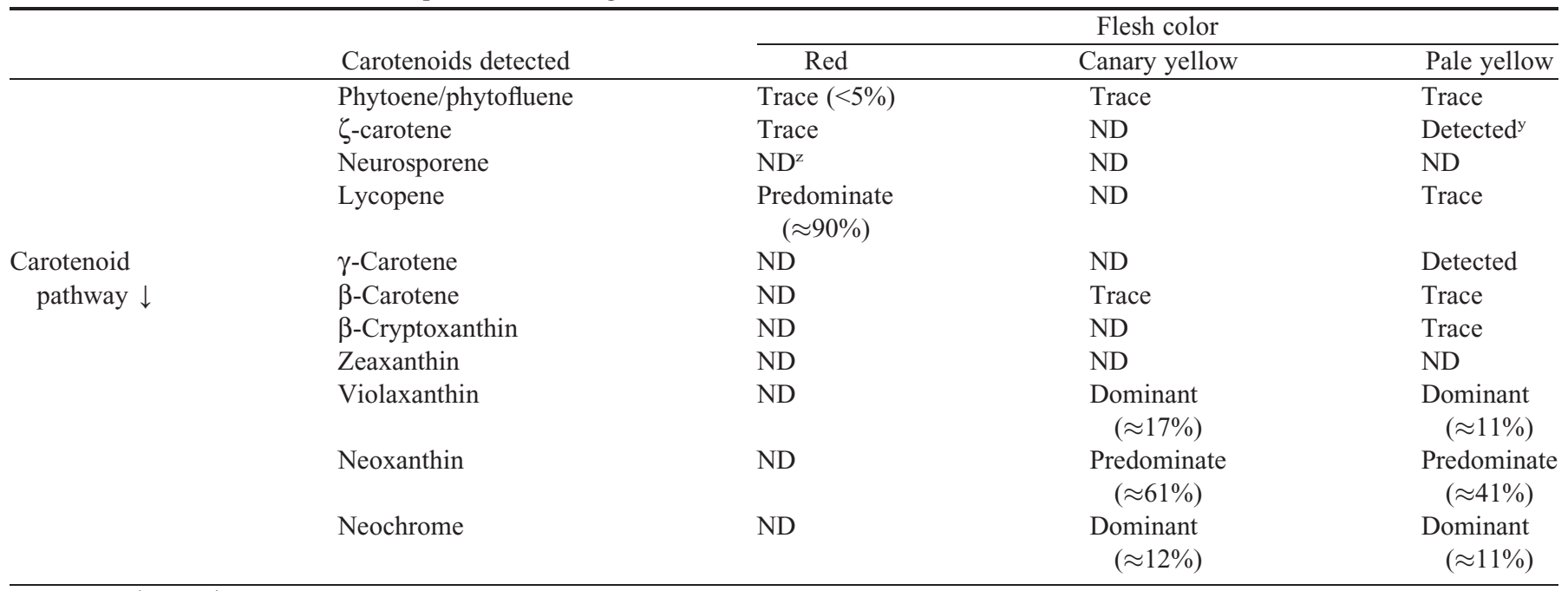

\footnotetext{
${ }^{\mathrm{z}} \mathrm{ND}=$ not detected.
}

${ }^{y}$ Small amount of carotenoid was detected in concentrated sample of pale yellow and may have been missed in other colors due to the lack of concentration of samples. 


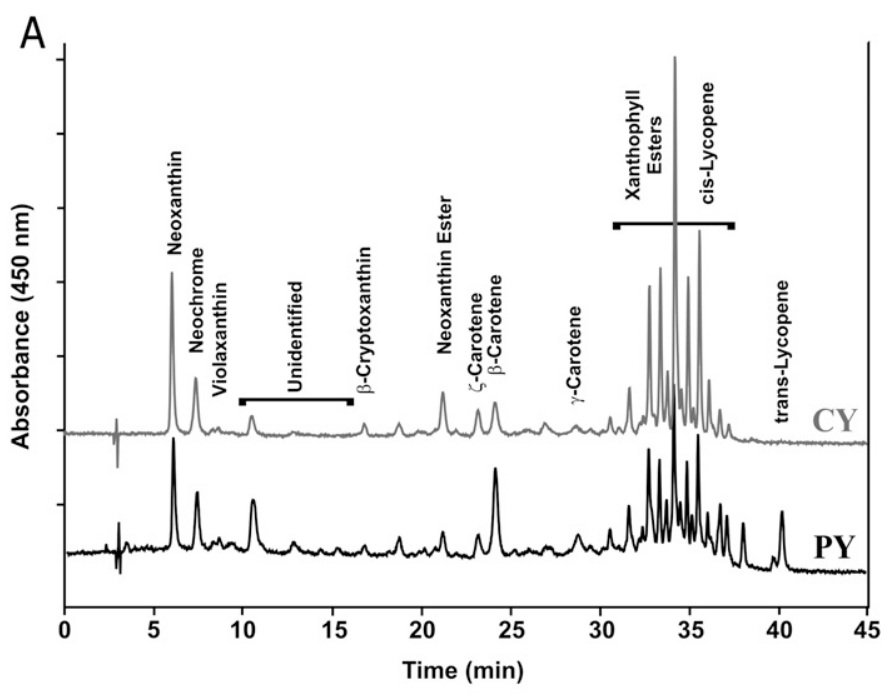

B

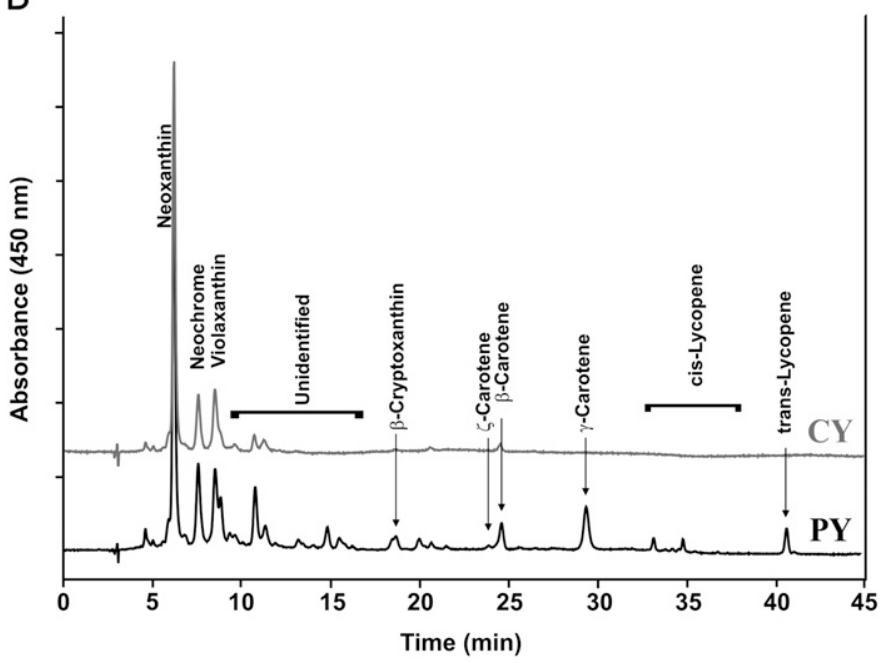

Fig. 2. High-resolution HPLC chromatograms of carotenoid profile before (A) and after (B) saponification in canary yellow (CY) and pale yellow (PY) watermelon fruit. Identified carotenoids are noted. Figure supplied by Craft Technologies (Wilson, NC).

immediately upstream and downstream of neoxanthin, respectively) as significant minor carotenoids. These carotenoids all have similar light yellow colors (Meléndez-Martínez et al., 2007) and likely impart all or most of the yellow color in canary yellow fruit. It is yet unclear why canary yellow and pale yellow fruit have such low total carotenoid content $(7.6 \%$ and $1.9 \%$ of red flesh in this study, respectively). A question that remains is that if the mechanisms are in place to produce large amounts of lycopene in red flesh genotypes, then why is there such a low concentration of carotenoids in canary and pale yellow? One possibility is a feedback inhibition caused by production of downstream carotenoids; in this case, the pathway is shut down with an accumulation of xanthophylls. Another possibility is that downstream carotenoids could be readily degraded into other compounds, such as aromatic volatiles or other end products, causing little accumulation of total carotenoids.

Our carotenoid profiles showed that neoxanthin is the predominant carotenoid in canary yellow and pale yellow, but canary yellow contained noticeably more neoxanthin than pale yellow $\left(1.66 \mu \mathrm{g} \cdot \mathrm{g}^{-1}\right.$ compared with $\left.0.29 \mu \mathrm{g} \cdot \mathrm{g}^{-1}\right)$. This, along with the dominance of canary yellow to pale yellow, suggests that the genetic difference may be a less functional gene in the xanthophyll pathway of the pale yellow genotypes. While this normally leads to an accumulation of upstream carotenoids, as is the case of lycopene in red watermelon, it may be that some intermediate xanthophyll compounds (e.g., zeaxanthin), which were not detected in any samples, are rapidly degraded and fail to accumulate to detectable amounts. This hypothesis is further substantiated by the fact that we were able to detect more upstream carotenoids in pale yellow than in canary yellow.

CAPS MARKER ANALYSIS. Over two hundred individual plants from the family 'Yellow Doll' $\times$ 'Sweet Princess', including both parents, $F_{1}, F_{2}, B C$ (both parents), and $F_{3}$ progenies, were genotyped (Tables 4 and 5) using our previously reported CAPS marker (Bang et al., 2007). The CAPS marker derived from the SNP Phe226 in the LCYB gene has perfectly cosegregated with flesh color in every case, which now includes over four hundred individuals, including our original population and additional breeding lines (data not shown). This combined data as well as the chi-square test of allele segregation (Table 4 ) confirmed that LCYB is the best candidate gene for the color determinant $C$ gene in watermelon. We created $F_{3}$ families from $12 \mathrm{~F}_{2}$ individuals and compared segregation patterns with our CAPS marker (Table 5). A total of six plants from each source was examined for LCYB genotype and color segregation. We observed that flesh color segregation matched the genotype of the $F_{2}$ according to our marker, where heterozygous plants continued to segregate for yellow and red flesh color, but homozygous plants did not segregate, except for canary yellow and pale yellow (assuming $P y$ was heterozygous).

Pale yellow phenotypes also contained a dominant LCYB allele in all cases. In addition, there are cultivars of watermelon characterized as having white flesh (e.g., 'Cream of Saskatchewan' and 'White Wonder'), but close examination reveals that they have a faint yellowish color indicative of trace amounts of carotenoids, which is distinct from the pure white flesh of citron watermelon that lack any detectable carotenoids (e.g., PI494531). We isolated genomic DNA from 'Cream of Saskatchewan' and compared sequences of the LCYB gene and determined that this cultivar carries the dominant LCYB allele (data not shown). Hence, the pale yellow phenotype requires a dominant $C$ (LCYB) for expression.

Table 4. Chi-square test of the segregation ratio between canary yellow and red alleles of watermelon lycopene $\beta$-cyclase (LCBY) singlenucleotide polymorphism (SNP) Phe226 in the $F_{2}$ and backcross progeny derived from a cross between 'Yellow Doll' (canary yellow) and 'Sweet Princess' (red).

\begin{tabular}{lcccc}
\hline Generation & $\begin{array}{c}\text { Observed (canary } \\
\text { yellow allele: } \\
\text { red allele) }\end{array}$ & $\begin{array}{c}\text { Expected (canary } \\
\text { yellow allele: } \\
\text { red allele) }\end{array}$ & $\chi^{2}$ & $P$ \\
\hline $\mathrm{F}_{2}$ & $59: 17$ & $3: 1$ & 0.28 & 0.60 \\
$\mathrm{BC}_{\text {Canary Yellow }}$ & $11: 0$ & $9: 7$ & 14.12 & $<0.01$ \\
$\mathrm{BC}_{\text {Red }}$ & $16: 22$ & $1: 0$ & 0 & 1 \\
& & $1: 1$ & 0.94 & 0.33 \\
& & $1: 3$ & 5.93 & 0.15 \\
\hline
\end{tabular}

${ }^{\overline{2} \text { Canary yellow allele is dominant LCYB where LCYB SNP Phe226 is }}$ TT for homozygous CY or TG for heterozygous CY; red allele is recessive LCYB where LCYB SNP Phe226 is GG for red. 
Table 5. Flesh color segregation and lycopene $\beta$-cyclase (LCYB) marker genotype analysis of $F_{3}$ progeny derived from a cross between 'Yellow Doll' (canary yellow) and 'Sweet Princess' (red) watermelon. Fruit colors were categorized into three colors: canary yellow (CY), pale yellow (PY), and red.

\begin{tabular}{llcc}
\hline & $\begin{array}{c}\text { Flesh color } \\
\text { of source } \mathrm{F}_{2}\end{array}$ & $\begin{array}{c}\text { LCYB genotype } \\
\text { of source } \mathrm{F}_{2}\end{array}$ & $\begin{array}{c}\text { Observed flesh color } \\
\text { segregation within } \mathrm{F}_{3} \\
(\mathrm{CY}: \mathrm{PY}: \mathrm{red})^{\mathrm{z}}\end{array}$ \\
\hline $\mathrm{F}_{3}$ & Canary yellow & LCYB/lcyb & $4: 0: 2$ \\
$\mathrm{~F}_{3}-01$ & CCYB/lcyb & $5: 0: 1$ \\
$\mathrm{~F}_{3}-02$ & Canary yellow & LCYB/LCYB & $5: 1: 0$ \\
$\mathrm{~F}_{3}-03$ & Canary yellow & LCY & $3: 2: 1$ \\
$\mathrm{~F}_{3}-04$ & Canary yellow & LCYB/lcyb & $3: 0: 3$ \\
$\mathrm{~F}_{3}-05$ & Canary yellow & LCYB/lcyb & $4: 0: 2$ \\
$\mathrm{~F}_{3}-06$ & Canary yellow & LCYB/lcyb & $5: 1: 0$ \\
$\mathrm{~F}_{3}-07$ & Canary yellow & LCYB/LCYB & $5: 0: 1$ \\
$\mathrm{~F}_{3}-08$ & Canary yellow & LCYB/lcyb & $0: 0: 6$ \\
$\mathrm{~F}_{3}-09$ & Red & $l c y b / l c y b$ & $0: 0: 6$ \\
$\mathrm{~F}_{3}-10$ & Red & $l c y b / l c y b$ & $0: 0: 6$ \\
$\mathrm{~F}_{3}-11$ & Red & $l c y b / l c y b$ & $0: 0: 6$ \\
$\mathrm{~F}_{3}-12$ & Red & $l c y b / l c y b$ &
\end{tabular}

${ }^{\mathrm{z}} \mathrm{A}$ total of six fruit was tested for segregation and genotyping analysis.

Functional analysis of our LCYB clones from red flesh and canary yellow flesh watermelon using lycopene producing Escherichia coli was recently carried out in a separate laboratory. The lycopene-producing $E$. coli colonies that were transformed with the canary yellow LCYB clone produced $\beta$-carotene, while colonies that were transformed with the red LCYB clone only produced lycopene (Yaakov Tadmor, personal communication).

This leads to a two-gene model that has previously been reported, but instead of the $C$ gene being influenced by an inhibitory gene $(i-C)$, we propose a $p y$ gene for pale yellow flesh in watermelon that is epistatic to $C$ where a recessive $p y$, in the presence of a dominant $C$, leads to the pale yellow phenotype. In this model, genotypes $C_{-} P y_{-}$have canary yellow flesh, genotypes $C_{\text {_ }}$ pypy are pale yellow, and $c c_{-}$, have red flesh.

\section{Conclusions}

The genetic models on flesh color inheritance proposed so far suggested that several genes are involved in flesh color determination of watermelon (Gusmini and Wehner, 2006; Henderson et al., 1998; Poole, 1944; Porter, 1937; Shimotsuma, 1963). Those findings relied solely on genetic studies and were not associated with carotenoid composition or molecular characterization. Also, previous studies on carotenoid biosynthesis were not linked to inheritance or molecular data. Because flesh color patterns were determined by visual evaluations and type lines for those colors are not readily available, flesh colors reported in previous studies may not be properly categorized. For example, it is difficult to distinguish between canary yellow and pale yellow when fruit are immature and it is also possible that pale yellow may have been miscategorized as white flesh because previous categorizations were based on visual color and lacked biochemical profiles.

This study links genetic data, biochemical analysis, and molecular characterization of genes and gene activity together to elucidate carotenogenesis in watermelon. We found the predominant carotenoid in canary yellow and pale yellow watermelon to be neoxanthin, and offer a new perspective in terms of extraction methodology and solvents compared with conventional protocols for carotenoid methodology in watermelon. We have demonstrated that a single gene determines canary yellow or red flesh in watermelon and have linked the $C$ gene in watermelon to LCYB in the carotenoid pathway. We have discovered a new, distinct flesh color (pale yellow) that has likely been misclassified in the past as white flesh, and we propose a single recessive gene $p y$ that operates in the presence of a dominant $C$ allele resulting in the pale yellow phenotype.

\section{Literature Cited}

Bang, H., S. Kim, D. Leskovar, and S. King. 2007. Development of a codominant CAPS marker for allelic selection between canary yellow and red watermelon based on SNP in lycopene $\beta$-cyclase (LCYB) gene. Mol. Breed. 20:63-72.

Bramley, P.M. 2000. Is lycopene beneficial to human health? Phytochemistry 54:233-236.

Craft, N.E. 2001. Chromatographic techniques for carotenoid separation. Curr. Protocols Food Anal. Chem. F2.3.1-F2.3.15.

Davis, A.R., J. Collins, W.W. Fish, Y. Tadmor, C.L. Webber Iii, and P. Perkins-Veazie. 2007. Rapid method for total carotenoid detection in canary yellow-fleshed watermelon. J. Food Sci. 72:S319-S323.

Fish, W.W., P. Perkins-Veazie, and J.K. Collins. 2002. A quantitative assay for lycopene that utilizes reduced volumes of organic solvents. J. Food Compost. Anal. 15:309-317.

Gerster, H. 1997. The potential role of lycopene for human health. J. Amer. Coll. Nutr. 16:109-126.

Giovannucci, E., E.B. Rimm, Y. Liu, M.J. Stampfer, and W.C. Willett. 2002. A prospective study of tomato products, lycopene, and prostate cancer risk. J. Natl. Cancer Inst. 94:391-398.

Gusmini, G. and T.C. Wehner. 2006. Qualitative inheritance of rind pattern and flesh color in watermelon. J. Hered. 97:177-185.

Henderson, W., G. Scott, and T. Wehner. 1998. Interaction of flesh color genes in watermelon. J. Hered. 89:50-53.

Henderson, W.R. 1989. Inheritance of orange flesh color in watermelon. Cucurbit Genet. Coop. Rpt. 12:59-63.

Hirschberg, J. 2001. Carotenoid biosynthesis in flowering plants. Curr. Opin. Plant Biol. 4:210-218.

Isaacson, T., G. Ronen, D. Zamir, and J. Hirschberg. 2002. Cloning of tangerine from tomato reveals a carotenoid isomerase essential for the production of $\beta$-carotene and xanthophylls in plants. Plant Cell 14:333-342.

Isaacson, T., I. Ohad, P. Beyer, and J. Hirschberg. 2004. Analysis in vitro of the enzyme CRTISO Establishes a poly-cis-carotenoid biosynthesis pathway in plants. Plant Physiol. 136:4246-4255.

Lewinsohn, E., Y. Sitrit, E. Bar, Y. Azulay, A. Meir, D. Zamir, and Y. Tadmor. 2005b. Carotenoid pigmentation affects the volatile composition of tomato and watermelon fruits, as revealed by comparative genetic analyses. J. Agr. Food Chem. 53:3142-3148.

Lewinsohn, E., Y. Sitrit, E. Bar, Y. Azulay, M. Ibdah, A. Meir, E. Yosef, D. Zamir, and Y. Tadmor. 2005a. Not just colors: Carotenoid degradation as a link between pigmentation and aroma in tomato and watermelon fruit. Trends Food Sci. Technol. 16:407-415.

Meléndez-Martínez, A.J., G. Britton, I.M. Vicario, and F.J. Heredia. 2007. Relationship between the colour and the chemical structure of carotenoid pigments. Food Chem. 101:1145-1150.

Perkins-Veazie, P., J.K. Collins, A.R. Davis, and W. Roberts. 2006. Carotenoid content of 50 watermelon cultivars. J. Agr. Food Chem. 54:2593-2597.

Poole, C.F. 1944. Genetics of cultivated cucurbits. J. Hered. 35:122-128. Porter, D.R. 1937. Inheritance of certain fruit and seed characters in watermelons. Hilgardia 10:489-509.

Rodriguez, G.A. 2001. Extraction, isolation, and purification of carotenoids. Curr. Protocols Food Anal. Chem. F2.1.1-F2.1.8.

Ronen, G., L. Carmel-Goren, D. Zamir, and J. Hirschberg. 2000. An alternative pathway to $\beta$-carotene formation in plant chromoplasts discovered by map-based cloning of Beta and old-gold color mutations in tomato. Proc. Natl. Acad. Sci. USA 97:11102-11107. 
Ronen, G., M. Cohen, D. Zamir, and J. Hirschberg. 1999. Regulation of carotenoid biosynthesis during tomato fruit development: Expression of the gene for lycopene $\varepsilon$-cyclase is down-regulated during ripening and is elevated in the mutant Delta. Plant J. 17:341-351.

Sauret-Güeto, S., P. Botella-Pavia, and M. Rodriguez-Concepción. 2003. Molecular tools for the metabolic engineering of carotenoid biosynthesis in plants. Recent Res. Dev. Plant Mol. Biol. 1:339-363.

Shimotsuma, M. 1963. Cytogenetical studies in the genus Citrullus. VI. Inheritance of several characters in watermelons. Jpn. J. Breeding $13: 235-240$.
Tadmor, Y., N. Katzir, S. King, A. Levi, A. Davis, and J. Hirschberg. 2004. Fruit coloration in watermelon: Lesson from the tomato. Cucurbitaceae 2004, the 8th EUCARPIA Meeting on Cucurbit Genetics and Breeding. Olomouc, Czech Republic. p. 181-185.

Tadmor, Y., S. King, A. Levi, A. Davis, A. Meir, B. Wasserman, J. Hirschberg, and E. Lewinsohn. 2005. Comparative fruit colouration in watermelon and tomato. Food Res. Int. 38:837-841.

Wehner, T.C. 2007. The gene list for watermelon. Cucurbit Genet. Coop. Rpt. 30:96-120. 